\title{
Impact of Automated Methods on Forensic Workflows
}

\author{
Kevin WP Miller*1 and Jennifer Duncan ${ }^{2}$ \\ ${ }^{1}$ Hamilton Robotics, 4970 Energy Way, USA \\ ${ }^{2}$ Texas Department of Public Safety, Houston Regional Crime Laboratory, USA
}

Submission: August 23, 2017; Published: August 28, 2017

*Corresponding author: Kevin WP Miller, Hamilton Robotics, 4970 Energy Way, Reno, Nevada USA, Tel: 775-544-5369;

Email: kevin.miller@hamiltoncompany.com

\begin{abstract}
Forensic sample testing backlogs and long turnaround times aren't just common laboratory problems; they potentially impact lives on both sides of the criminal offense. Mediating these issues by means of increasing laboratory personnel or overtime hours, or outsourcing to third-party testing labs, is often cost-prohibitive and not sustainable. At the same time, manual testing methods risk user variability and processing errors that may require reworking precious evidentiary samples. Automated methods provide increased sample throughput while decreasing active labor involvement, and also increase accuracy, precision and standardization of results. Additionally, by implementing automated solutions in the context of anall-encompassing overhaul of the laboratory's workflow process, forensic laboratories can maximize efficiency throughout the evidentiary sample life cycle to further reduce backlogs and increase turnaround times. Here, we illustrate a method whereby a pre-PCR DNA typing workflow was mapped, areas of inefficiency were identified, and improvements were incorporated into an automated workflow to achieve higher sample throughput and quality with fewer analysts and reduced need for repeat sample processing.
\end{abstract}

Keywords: Laboratory management, Automation, DNA typing, Backlog, Turn-around time, Quality metrics

Abbreviations: CODIS: Combined DNA Index System; DNA: Deoxyribonucleic Acid; DPS: Department of Public Safety; LIMS: Laboratory Information Management System; PCR: Polymerase Chain Reaction; TAT: Turn Around Time

\section{Introduction}

Despite federal grant programs and concerted efforts, sample testing backlogs and long turnaround times (TAT) remain burdensome for forensic labs. Contributing factors include new crimes committed, regulations constraining the time that untested evidence may remain within a laboratory, assay chemistry advances that present opportunities for the further testing of cold cases, and more. Traditionally, forensic administrators have tried to address backlog and improve turnaround by obtaining additional personnel, outsourcing casework, rejecting evidence obtained from certain types of cases such as high volume non-violent crimes, and adopting productivity methodologies such as Lean Six Sigma. Backlogs and long TATs aren't issues isolated to the forensic laboratory; instead, they create a ripple effect throughout the legal system, and may contribute to additional stress and hardships on both sides of the criminal offense as victim and defendant, if known, are forced to wait for results.

\section{Labor as a Method to Counter Backlogs}

Efficient use and expansion of human resources have been employed by many forensic administrators in the attempt to address backlogs and improve turnaround times within their facilities. Personnel are the most expensive commodity within a crime laboratory; however, they are also a substantial amount of a laboratory's already stretched-thin budget. Due to some government employment guidelines, providing analysts with overtime is a "zero sums" game, because employees often opt for compensatory time in lieu of pay. In these circumstances, time is leveraged and this means less labor hours available to work future cases. The consistent use of overtime only exacerbates backlog and turnaround woes. Alternatively, expanding the numbers of analysts in a laboratory drives budgetary costs up, and this is routinely resisted by governments that are responsible for personnel-related costs well beyond the years that an individual serves as an analyst. Training new hires, especially in the analytical disciplines, requires a large investment of 
time and money. Senior criminalists, the most expensive of the laboratory's human resources, typically mentor newly onboarded criminalists. This diverts the most experienced people in the laboratory away from otherwise working the most difficult and challenging cases in the lab. In short, trying to solve backlog and turnaround issues using personnel alone further taxes an already over burdened system.

Outsourcing case work to contract laboratories is a way for government laboratories to obtain additional human resources without incurring overhead expenses. However, this solution has downsides of its own. Evidence must still be processed to the point where documented samples can be sent out, for example, and both the contractors and their work product must be assessed and internally verified. Needless to say, performing contractor and casework oversight is often as burdensome to a laboratory as if the casework were originally done inhouse. Additionally, the need for in-house review of contracted casework before a final report of results is issued can actually add to the number of days that it takes the forensic laboratory to complete a case. Oftentimes those downstream of the laboratory, which outsourcing was intended to help, continue to wait.

Laboratory administrators may develop policies not to work some types of cases in order to manage backlogs and turnaround times. DNA sample submissions from high volume property crimes, for example, may be rejected in favor of directing laboratory resources toward more serious violent crimes as a means of resource management despite the obvious payoffs of entering these results into databases such as the Combined DNA Index System (CODIS) in order to develop investigative leads that would not otherwise be available. These policy decisions are felt throughout the justice system, and also to the victims and the communities in which they live.

\section{The Impact of Manual Processing}

Regardless of whether testing is done in-house or outsourced, and often independent of skill or experience, manual sample testing is limited in throughput, subject to variability, and vulnerable to errors that can risk accuracy, precision and reproducibility. Active analyst involvement is the rate limiting factor in the number of samples that can be processed at one time. Analyst variability may also be a concern. Individual performance may vary due to stress and fatigue, especially when working overtime, whereas variability between individuals may arise from differences in laboratory technique, and approach to subjective criteria such as visual determinations. Assay incubation periods can add to variability and reduce reproducibility. This can occur when competing for use of shared instrumentation, or when analysts are interrupted to attend to other tasks.

In addition to variability, manual analyst errors, including manual transcription errors and cross-contamination, may result in the need to retest an evidentiary sample, which exacerbates backlog and TAT issues. If results are successfully challenged in court, then an analyst's reliability may be called into question for the remainder of their career. In cases of intentional error such as "dry-labbing", the reputation of both the individual analyst and the entire laboratory are at stake and individuals risk losing their jobs or facing criminal prosecution if caught. Checkpoints are often put in place throughout the sample life cycle to prevent quality issues, but this often forsakes time and throughput issues, as analysts are interrupted to conduct these quality reviews.

\section{Presenting the Case for Automated Forensic Testing}

In highly regulated, resource constrained environments, laboratory robotics such as automated liquid handlers can bring efficiencies and robust quality of results to sample preprocessing and processing workflows without increasing labor costs, to ease testing bottlenecks and allow the judicial process to continue without delay. At the same time, these benefits are further enhanced by conducting an all-encompassing assessment of the entire case ownership chain, from sample collection and retrieval through processing and data analysis, to final report writing.

To illustrate this point, the Texas Department of Public Safety (DPS) Crime Laboratory in Houston, Texas, previously employed an EZ1 robotic workstation (QIAGEN, Germantown, MD) for the automated purification of nucleic acids and a Freedom EVO® 150 workstation (Tecan, Männedorf, Switzerland) for amplification set-up. However, these instruments only addressed one portion of the overall DNA profiling workflow or were not widely adopted. Therefore, bottlenecks in the pre-PCR workflow could not be addressed using these instruments. The laboratory sought to streamline this workflow and also alleviate timeconsuming pinch-points throughout the entire case ownership chain. The laboratory added a Microlab ${ }^{\circledR}$ AutoLys STAR plus liquid handling workstation Hamilton Robotics, Reno, NV with integrated VENUS software to replace or encompass single-step instruments. The AutoLys STAR plus enabled batched parallel processing to increase throughput compared to manual methods when extracting biological stains from various substrates, even among different sample types. Whereas the DPS Laboratory incurred time and expense to validate their automated sample workflow, it is worth noting that these efforts could be considerably lessened by implementing vendor qualified forensic application solutions.

In addition to increasing the number of samples processed per analyst by up to $15 \%$ compared to manual methods, the integrated, batched workflow reduced quality-related incidents by $42 \%$ (Table 1 ). The number of samples in the automated workflow that required re-processing as a result of a quality incident decreased by 63\% (Table 2), thereby saving time, materials cost and evidence consumption. The decrease in quality events can be directly attributed to the sample traceability and 
process standardization offered by the AutoLys STAR plus. While the laboratory chain of custody was manually input into the laboratory information management system (LIMS), the use of AutoLys tubes imprinted with 2D barcodes created a chain of custody such that each sample is automatically and independently verified, and that the sample was correctly correlated with both its case and the assigned analyst. This reduced labor associated with manually verifying each sample, and also eliminated risk of analyst transcription errors. Additionally, the 2D bar-coding was used when automatically generating work lists for the liquid handling platform, thus further reducing time and transcription errors associated with manual methods. The AutoLys tubes were also automatically capped and uncapped only as necessary to reduce analyst involvement and reduce risk of contamination.

Table 1: Comparison of manual (2013) and automated (2016) laboratory workflow quality incidents. Automated liquid handling completely eliminated sample-related quality issues, for savings in time, money, and evidence consumption.

\begin{tabular}{|c|c|c|}
\hline Root Cause of Quality Incident & $\mathbf{2 0 1 3}$ & $\mathbf{2 0 1 6}$ \\
\hline${ }^{A}$ Analyst Method Set-Up Error & 5 & 0 \\
\hline Analyst Transcription Error & 2 & 7 \\
\hline${ }^{B}$ Sample Contamination - Manual Process & 17 & 0 \\
\hline Sample Contamination - Robotic Process & 0 & 0 \\
\hline Sample Discard (Accidental) & 1 & 0 \\
\hline Sample Plate Mis load (Amplification or CE) & 8 & 3 \\
\hline Sample Switch & 2 & 15 \\
\hline Other Error Type & 36 & \\
\hline
\end{tabular}

${ }^{A}$ Method set-up errors were analyst-related incidents that included improper placement of 96-well plates, a CE plate in backwards, use of improper tip types, using expired reagents, etc.

${ }^{B}$ All contamination events were considered to be the result of human error, even if the source of the contamination remained unresolved.

Table 2: Comparison of manual (2013) and automated (2016) sample re-processing.

\begin{tabular}{|c|c|c|}
\hline Re-Work Resulting from Quality Incident & $\mathbf{2 0 1 3}$ & $\mathbf{2 0 1 6}$ \\
\hline Re-Extraction & 17 & 2 \\
\hline Re-Amplification & 6 & 2 \\
\hline Re-Typing (CE) & 7 & $\mathbf{1 1}$ \\
\hline Total Number of Re-Works & $\mathbf{3 0}$ & 2 \\
\hline
\end{tabular}

The instrument's lock-out protocols prevented unauthorized or accidental changes, thus further increasing standardization and repeatability. The automated liquid handler incorporated pre-programmed pipetting and sample manipulation steps of the pre-PCR workflow to reduce active analyst time, reduce fatigue associated with pipetting, and remove the risk of crosscontamination or analyst error and variability. LIMS integration enabled automatically generated work lists that further reduced analyst time and risk of manual error. Once samples were processed, all data and records were digitally stored in the LIMS and output in a consistent, standardized manner, independent of the analyst. This information was used when creating workflows for other devices, reducing time spent manually creating additional lists. Additionally, it created standardized and detailed case files for streamlined technical review; creating efficiencies in the laboratory beyond that of the sample processing workflow itself, thus contributing to a reduction in turnaround times. While the automated system generated more detailed information in the reports for discovery, the reports contained fewer case- and analyst-related quality disclosures.

As fixed labor costs comprise a significant portion of the forensic laboratory budget, the ability to reallocate analysts to other activities while concurrently increasing the number of samples processed, and while decreasing quality incidents, were key benefits of the integrated, automated system (Table 3 ). Instrument use reduced active labor time, and freed forensic analysts to refocus their efforts on data interpretation and case report writing. Although reductions in turnaround time were not directly observed, the laboratory noted that it also absorbed a high degree of change during this time, including analyst turnover, new hire trainings, re-training of existing analysts, an increased number of validations brought about by industry mandates to move to new DNA typing kit chemistries, and advisory board approvals of validation work. 
Table 3: Since automated workflows were implemented, the laboratory has faced significant challenges, including personnel turn-over and special projects e.g. new kit validation, etc. These challenges notwithstanding, significantly more samples are processed with fewer analysts and lower quality incidents compared to manual methods.

\begin{tabular}{|c|c|c|}
\hline $\begin{array}{c}\text { Performance } \\
\text { Metrics }\end{array}$ & $\mathbf{2 0 1 3}$ & $\mathbf{2 0 1 6}$ \\
\hline $\begin{array}{c}\text { Number of DNA } \\
\text { Analysts }\end{array}$ & 16 & 11 \\
\hline $\begin{array}{c}\text { Number of Samples } \\
\text { Processed/Analyst }\end{array}$ & 462 & 533 \\
\hline $\begin{array}{c}\text { Number of Quality } \\
\text { Incidents/Analyst }\end{array}$ & 2.25 & 1.36 \\
\hline
\end{tabular}

\section{Conclusion}

While the obvious advantage of automated processing includes higher sample throughput with reduced costly labor involvement, additional benefits include greater efficiencies and quality results, especially in the context of an all-encompassing assessment of the overall workflow. The Texas Department of Public Safety Crime Laboratory is one example of how a fully integrated automated system can ease bottlenecks, increase quality of repeatable results, and create time-savings in their pre-PCR workflow. By incorporating laboratory automation into workflows, productivity and robustness are maximized so that the judicial process is not impinged by forensic laboratory backlogs and turnaround times.

\section{Conflict of Interest}

Kevin Miller is employed full-time by Hamilton Robotics as the Scientific Leader for Forensic Markets throughout Asia Pacific (less China) and the Americas. Hamilton Robotics manufactures and sells automated liquid handling platforms such the one used by the Texas Department of Public Safety, Houston Regional Crime Laboratory, as discussed in this paper.

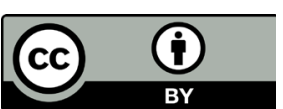

This work is licensed under Creative Commons Attribution 4.0 License DOI: $10.19080 /$ JFSCI.2017.04.555645

\section{Your next submission with Juniper Publishers will reach you the below assets}

- Quality Editorial service

- Swift Peer Review

- Reprints availability

- E-prints Service

- Manuscript Podcast for convenient understanding

- Global attainment for your research

- Manuscript accessibility in different formats ( Pdf, E-pub, Full Text, Audio)

- Unceasing customer service

Track the below URL for one-step submission https://juniperpublishers.com/online-submission. 